\title{
Coleta de secreção traqueal: estudo comparativo de técnicas
}

\author{
Silvia Dubou Serafim¹, Juliana Alves Souza1', Janice Cristina Soares ${ }^{1}$, Nara Lucia Frasson Dal Forno²
}

\begin{abstract}
RESUMO
Objetivo: Comparar, por meio de análise microbiológica, duas técnicas de coleta de secreção traqueal. Metodologia: estudo experimental, quantitativo, comparativo, cego, randomizado, desenvolvido na Unidade de Tratamento Intensivo (UTI). Foram realizadas duas técnicas de coleta em cada paciente hipersecretivo, entubado ou traqueostomizado, que necessitou de análise de secreção, sendo a ordem das mesmas aleatória. Na técnica 1 a sonda de aspiração foi cortada com tesoura estéril e colocada em pequenas parte dentro de frasco estéril. Já na técnica 2, a secreção que estava dentro da sonda de aspiração foi empurrada por fluxômetro de oxigênio para dentro do frasco estéril. Resultados: Os microorganismos mais encontrados na UTI foram Pseudomonas aeruginosa (13,64\%), Acinetobacter baumanni (13,64\%), e Candida spp (18,18\%). A relação entre ambas às técnicas foi de igualdade (coeficiente Kappa), tanto para a De Gram como para a identificação das bactérias. Conclusão: A análise microbiológica demonstrou que as técnicas de coleta de secreção traqueal com a sonda cortada e com o fluxo de oxigênio são equivalentes. Logo ambas podem ser utilizadas na rotina hospitalar.
\end{abstract}

Descritores: Infecção Hospitalar; Respiração Artificial; Análise Microbiológica.

\section{Collect tracheal secretion: a comparative study of techniques}

\begin{abstract}
Objective: The objective of this study is to compare, by means of microbiological analysis, two techniques of tracheal aspirates in intubated or tracheostomized patients. Methodology: The study was conducted from May to June 2012, in HUSM at UFSM, becoming an experimental study, qualitative and quantitative, comparative trial. There were two data collection techniques in each hipersecretive patient, intubated or tracheostomized, requiring analysis of secretion, with the same random order. In technique 1 the secretion was aspirated until probe was with a sufficient amount of secretion, after the aspiration tube was cut with sterile scissors, into small pieces within the sterile flask. In technique 2 , when the probe had a sufficient amount of secretion, it was clamped, the extender was disconnected and connected to the suction end of the oxygen flow meter. Following, the probe tip was placed into a sterile collection vial while the flow meter was connected to $15 \mathrm{I} /$ min so that the secretion present inside the tube is pushed into the pot. Results: The bacteria most commonly found in the Intensive Care Unit were Pseudomonas aeruginosa (13.64\%) and Acinetobacter baumannii (13.64\%) also had the presence of Candida spp (18.18\%). The relationship between both techniques is equal (Kappa coefficient), both as a Gram stain for bacteria. Conclusion: The microbiological analysis showed that the collection techniques with the tracheal secretion tube and cut the flow of oxygen are equivalent. Soon both can be used in hospital routine.
\end{abstract}

Descriptors: Infection; Artificial Respiration; Microbiological Analysis.

${ }^{1}$ Fisioterapeuta pela Universidade Federal de Santa Maria (UFSM), Santa Maria, RS, Brasil.

2 Farmacêutica pela Universidade Federal de Santa Maria (UFSM), Santa Maria, RS, Brasil. 


\section{Introdução}

Atualmente denominada infecção relacionada à assistência à saúde (IRAS), a infecção hospitalar (IH), é toda a infecção adquirida em um prazo de 48 a 72 horas após a internação hospitalar e que não esteja em seu período de incubação ${ }^{1}$.

Nas UTIs a pneumonia associada à ventilação mecânica (PAV) é a infecção mais frequente, ocorrendo em 9 a $27 \%$ de todos pacientes entubados por mais de 48 horas $^{2}$. Isto porque, a ventilação mecânica é um dos principais fatores de risco para desenvolvimento de pneumonia. A PAV aumenta o risco de óbito, prolonga o tempo de ventilação mecânica e de internação, aumentando, também, os gastos com o tratamento ${ }^{3}$.

A precocidade do diagnóstico e o tratamento adequado são fundamentais para o sucesso de condução dos casos de PAV. Ter o diagnóstico etiológico é o ideal, bem como o perfil de sensibilidade do patógeno aos antibióticos disponíveis. Dessa forma, o tratamento pode ser apropriado, sem riscos de não tratar o agente envolvido, sem a necessidade de se estender muito o espectro, o que poderia trazer, com o tempo, resistência aos antibióticos administrados ${ }^{4}$. No entanto, este ideal não é facilmente alcançado na PAV. Os sintomas clínicos são ambíguos, não há um padrão ouro para o diagnóstico da mesma e a mortalidade ainda é alta, em torno de $30-40 \%{ }^{5}$.

O correto diagnóstico etiológico só pode ser realizado com isolamento do agente a partir do material não contaminado por possíveis bactérias colonizadoras como o líquido pleural, sangue ou aspirado de punção pulmonar transtorácica. O diagnóstico etiológico da PAV tem-se tentado por meio de culturas de secreções respiratórias ${ }^{6}$.

As técnicas de obtenção da secreção respiratória podem ser invasivas como BAL (lavado broncoalveolar), PSB (escovado brônquico com cateter protegido) e não invasivas como aspiração da secreção traqueal, Mini BAL (Mini Lavado Broncoalveolar). Porém, somente a cultura desses materiais não permite o diagnóstico correto de PAV, visto que a colonização bacteriana nesses pacientes é comum. A presença de um novo e persistente infiltrado na radiografia de tórax acompanhada de secreção traqueal purulenta, febre ou hipotermia, leucocitose ou leucopenia ajudam a fechar o diagnóstico ${ }^{5}$.

Tendo em vista esses critérios o diagnóstico microbiológico é uma importante ferramenta para adequar a terapia antimicrobiana e diminuir a mortalidade associada à PAV ${ }^{7}$. Sabe-se que a coleta e/ou transporte inadequado da amostra de secreção traqueal pode ocasionar falhas no isolamento do agente etiológico causador da infecção, favorecer 0 desenvolvimento da flora contaminante habitual, induzindo a um tratamento inapropriado. Portanto, a qualidade do resultado liberado pelo laboratório está diretamente relacionada à qualidade da amostra recebida ${ }^{8}$.

A coleta de secreção traqueal é um procedimento muito utilizado nas UTIs, porém não existe comprovação de uma técnica adequada, nem padronização descrita na literatura, para sua realização. Observa-se, na prática clínica, que cada profissional faz a seu modo, entretanto se a coleta for inadequada, o resultado final será inadequado. As infecções do trato respiratório inferior incluem um grande número de etiologias e o diagnóstico microbiológico dessas infecções é frequentemente prejudicado pela contaminação da amostra durante a coleta. Considerando estes aspectos, o objetivo desse estudo foi comparar por meio de análise microbiológica, duas técnicas de coleta de secreção traqueal em pacientes entubados ou traqueostomizados.

\section{Metodologia}

O estudo foi desenvolvido em um hospital universitário, de maio a julho de 2012, após a aprovação pelo Comitê de Ética CAE 01314912.0.0000.5346, respeitando os princípios éticos estabelecidos pela resolução do Conselho Nacional de Saúde, constituindo-se de um estudo experimental, quantitativo, comparativo, cego, randomizado.

\section{Seleção da amostra}

Foram selecionados 22 pacientes internados na Unidade de Tratamento Intensivo (UTI), mediante assinatura de um responsável do Termo de Consentimento Livre e Esclarecido. Pacientes de ambos os sexos, em via aérea artificial, hipersecretivos, que necessitassem de coleta de secreção traqueal por prescrição médica, durante o período destinado a pesquisa, foram incluídos no estudo. Foram excluídos os pacientes que, não estivessem fazendo uso de via aérea artificial, que necessitassem de sistema fechado de aspiração traqueal e, também, indivíduos com sangramento das vias aéreas inferiores. 


\section{Coleta de secreção traqueal}

Foram realizadas duas técnicas, denominadas técnica 1 e técnica 2. Na técnica 1 após a higienização das mãos e colocação de luvas estéreis introduziu-se uma sonda de aspiração estéril de calibre adequado já conectada ao sistema de vácuo, através do tubo endotraqueal até encontrar resistência; aspirou-se até que a sonda estivesse com uma quantidade suficiente de secreção (volume mínimo de $1 \mathrm{ml}$ ); cortou-se a sonda de aspiração com tesoura estéril em pequenas partes dentro do frasco estéril. Na técnica 2 após higienização das mãos e colocação de luvas estéreis, introduziu-se uma sonda de aspiração estéril de calibre adequado conectada ao sistema de vácuo, através do tubo endotraqueal até encontrar resistência; quando a sonda de aspiração estava com uma quantidade suficiente de secreção a mesma foi clampeada e desconectada do extensor de aspiração; na sequência foi acoplada a extremidade do fluxômetro de oxigênio e a ponta da sonda colocada dentro do pote estéril de coleta; o fluxômetro foi acionado a $15 \mathrm{l} / \mathrm{min}$ fazendo com que a secreção presente no interior da sonda fosse empurrada para dentro do pote. Em ambas as coletas não foi instilada qualquer solução.

As duas técnicas de coleta foram randomizadas de forma aleatória, sendo a primeira coleta com a técnica um e a segunda com a técnica dois e depois fazendo-se o inverso e assim sucessivamente. As amostras foram coletadas logo após o atendimento fisioterapêutico, pelo turno da manhã, sempre pelo mesmo técnico de enfermagem, que recebeu um treinamento antes de começar o processo de coletas.

\section{Análise microbiológica}

Após a coleta, o material, devidamente identificado, foi imediatamente encaminhado ao Laboratório de Análises Clínicas (LAC) de um Hospital Universitário para análise microbiológica. A seguir as amostras foram interpretadas por uma farmacêutica.

No LAC, inicialmente, foi retirado todo o material da sonda (Técnica 1) e do frasco estéril (Técnica 2), colocando uma parte em lâminas. Foi usada a parte mais purulenta de cada amostra e, posteriormente, foi feita a leitura microscópica. Nessa leitura foram observados os critérios de Oplustil (2010) para a aceitabilidade, sendo consideradas as amostras que tiveram $\leq 10$ células epiteliais/campo e $\geq 25$ leucócitos/campo da secreção. Na sequência, as amostras foram colocadas nos meios Ágar sangue, Ágar Azida ou Ágar McConkey, sendo realizada a semeadura por esgotamento em três sentidos. Nas amostras em que houve um crescimento bacteriano superior a $105 \mathrm{UFC} / \mathrm{ml}$ realizou-se a incubação $35^{\circ} \mathrm{C} \pm 2{ }^{\circ} \mathrm{C}$ em atmosfera adequada durante 24 a 48 horas. Naquelas em que 0 crescimento bacteriano foi inferior a $105 \mathrm{UFC} / \mathrm{ml}$ não foi dado andamento à análise.

\section{Análise dos dados}

O software STATSOFTSTATISTICA 7.1. e o BioEstat 5.0 foram utilizados para as análises, bem como estatística descritiva para apresentação das frequências encontradas. A comparação entre as técnicas 1 e 2 quanto à análise microbiológica foi realizada, num primeiro momento, a partir da avaliação da concordância ou não entre os resultados, ou seja número de casos cujos resultados foram iguais e diferentes, calculado pelo Coeficiente de Kappa (ponderado). Esta medida de concordância tem como valor máximo 1 que representa total concordância e os valores próximos e até abaixo de 0 que indicam nenhuma concordância, ou que a concordância foi exatamente a esperada pelo acaso; mas seu valor não tem interpretação como intensidade de discordância. Logo valores de Kappa < 0 indicam nenhuma concordância, de 0 a 0,39 concordância leve; 0,40 a 0,79 moderada e de 0,80 a 1 concordância quase perfeita ${ }^{11}$. Num segundo momento o Teste do Qui-quadrado e Exato de Fisher foram utilizados para comparação das frequências de microorganismos encontrados e da coloração Gram dos mesmos entre as técnicas 1 e 2 . Admitiu-se um nível de significância de $5 \%$ em todos os testes $(p<0,05)$.

\section{Resultados}

A análise microbiológica demostrou que os germes mais prevalentes nos indivíduos avaliados foram o Acinetobacter baumanni presente em 13,64\% (3/22), a Pseudomonas aeruginosa em 13,64\% (3/22) e a Candida spp presente em $18,18 \%(4 / 22)$ dos mesmos, conforme exposto na Tabela 1. 
Tabela 1 - Germes mais frequentes na Unidade de Terapia Intensiva do Hospital ... - análise microbiológica.

\begin{tabular}{c|c|c}
\hline \multirow{2}{*}{ Germes } & \multicolumn{2}{c}{$\begin{array}{c}\text { Pacientes } \\
(\mathrm{n}=22)\end{array}$} \\
\cline { 2 - 3 } & $\mathrm{n}$ & $\%$ \\
\hline Achromobacter xylosoxidans & & \\
\hline Acinetobacter baumanni & 1 & 4,55 \\
\hline Beta lactamase positiva & 3 & 13,64 \\
\hline Candida spp & 1 & 4,55 \\
\hline Coagulase-negative Staphylococcus species & 1 & 4,55 \\
\hline Klebsiella pneumoniae & 1 & 4,55 \\
\hline Moraxella catarrhalis & 2 & 9,09 \\
\hline Pseudomonas aeruginosa & 3 & 13,64 \\
\hline Serratia marcescens & 2 & 9,09 \\
\hline Staphylococcus aureus & 1 & 4,55 \\
\hline Stenotrophomonas maltophilia & 1 & 4,55 \\
\hline
\end{tabular}

A frequência dos germes da Unidade de Terapia Intensiva foram classificados conforme a classificação De Gram. 0 estudo demonstra que há um predomínio de 59,09\% dos germes Gram negativos. Já os De Gram positivo aparecem em $13,64 \%$ dos pacientes. Em alguns pacientes aparecem ambos De gram, cerca de $9,09 \%$. Em quatro pacientes $(18,18 \%)$ não aparece nenhum microorganismo.

Em relação à qualidade das amostras microbiológicas, todas (técnica 1 e 2) atenderam aos critérios de Opustil ${ }^{10}$, uma vez que o LAC não solicitou a repetição de nenhuma coleta.

Acomparação entre as técnicas de coleta de secreção traqueal quanto ao número de células, leucócitos, microorganismos e De Gram está demostrada na Tabela 2. Observa-se que a porcentagem de exames com resultados iguais foi sempre maior, com uma concordância fraca quanto ao número de leucócitos e forte quando aos germes encontrados.

Tabela 2.- Grau de concordância entre as técnicas 1 e 2 de coleta de secreção traqueal quanto à análise microbiológica.

\begin{tabular}{c|c|c|c|c|c|c}
\hline Análise Microbiológica & \multicolumn{2}{|c|}{ técnicas 1 e 2 ( $=22$ pacientes) } & \multicolumn{2}{l}{ Coeficiente de concordância } \\
\hline & \multicolumn{2}{|c|}{ Igual } & \multicolumn{2}{c|}{ Diferente } & Kappa & Interpretação \\
\hline $\mathrm{N}^{0}$ de Células & 16 & 72,73 & 6 & 27,27 & 0,48 & Moderada \\
\hline $\mathrm{N}^{0}$ de Leucócitos & 12 & 54,55 & 10 & 45,45 & 0,27 & Leve \\
\hline Microorganismos & 17 & 77,27 & 5 & 22,73 & 0,69 & Forte \\
\hline De Gram & 15 & 68,18 & 7 & 31,82 & 0,54 & Moderada \\
\hline
\end{tabular}

Na Tabela 3 a comparação específica dos microorganismos que cresceram nas coletas realizadas pela técnica 1 e 2 está demonstrada. Não foram verificadas diferenças estatísticas entre as técnicas, embora em cinco indivíduos houvesse crescimento diferente dos germes Serratia marcescens, Candida spp, Staphylococcus aureus e Coagulase-negative Staphylococcus species. 
Tabela 3.- Comparação entre as técnicas 1 e 2 de coleta de secreção traqueal quanto ao crescimento microbiano.

\begin{tabular}{c|c|c|c|c|c}
\hline \multirow{2}{*}{ Microorganismos } & \multicolumn{2}{|c|}{ Técnica 1 } & \multicolumn{2}{c|}{ Técnica 2 } & \multirow{2}{*}{ p - valor } \\
\cline { 2 - 5 } & $\mathrm{n}$ & $\%$ & $\mathrm{n}$ & $\%$ & \\
\hline Achromobacter xylosoxidans & 1 & 4,55 & 1 & 4,55 & 1 \\
\hline Acinetobacter baumanni & 3 & 13,64 & 3 & 13,64 & 1 \\
\hline Beta lactamase positiva & 1 & 4,55 & 1 & 4,55 & 1 \\
\hline Candida spp & 4 & 9,09 & 4 & 18,11 & 1 \\
\hline Coagulase-negative S. species & 1 & 9,09 & 0 & 0 & - \\
\hline Klebsiella pneumoniae & 1 & 4,55 & 1 & 4,55 & 1 \\
\hline Moraxella catarrhalis & 2 & 4,55 & 1 & 4,55 & 1 \\
\hline Pseudomonas aeruginosa & 3 & 13,64 & 3 & 13,64 & 1 \\
\hline Serratia marcescens & 2 & 9,09 & 1 & 4,55 & 1 \\
\hline Staphylococcus aureus & 1 & 4,55 & 0 & 0 & - \\
\hline Stenotrophomonas maltophilia & 1 & 4,55 & 1 & 4,55 & 1 \\
\hline
\end{tabular}

Teste Qui Quadrado/Teste Exato de Fisher, $p<0,05 . n=22$ pacientes

A comparação entre as técnicas quanto à coloração De Gram está na tabela 4, também não foram encontradas diferenças estatísticas.

Tabela 4. Comparação entre as técnicas 1 e 2 de coleta de secreção traqueal quanto a coloração De Gram os microorganismos.

\begin{tabular}{c|c|c|c|c|c}
\hline Coloração Gram & \multicolumn{2}{|c|}{ Técnica 1 } & \multicolumn{2}{|c|}{ Técnica 2 } & \multirow{2}{*}{ - valor } \\
\cline { 2 - 5 } & $\mathrm{n}$ & $\%$ & $\mathrm{n}$ & $\%$ & \\
\hline Positivo & 2 & 9,09 & 1 & 4,55 & 1,00 \\
\hline Negativo & 12 & 54,55 & 10 & 45,45 & 0,54 \\
\hline Ambos & 2 & 9,09 & 0 & 0,00 & - \\
\hline
\end{tabular}

Teste Qui Quadrado/Teste Exato de Fisher, $p<0,05 . n=22$ pacientes

\section{Discussão}

Não há uma padronização descrita na literatura quando à técnica ideal para coleta de secreção das vias aéreas inferiores. Entretanto, com uma técnica mais apurada é mais fácil diagnosticar a patologia, ajudando a ajustar a medicação e diminuir o tempo de internação e a mortalidade hospitalar ${ }^{10}$.

Nesse estudo duas dessas técnicas foram comparadas quanto à frequência de bactérias, De Gram, número de leucócitos e número de células. O resultado estatístico não demonstrou diferenças significativas entre as mesmas.

As amostras foram analisadas na forma qualitativa, pois no LAC do hospital essa é a rotina de análise microbiológica, assim como na maioria das UTIs, onde o diagnóstico de PAV é clínico e a identificação do agente é realizada por meio de análise qualitativa ${ }^{9}$. Embora atualmente se preconize a análise quantitativa para estabelecer a presença de $\mathrm{PAV}^{7}$, a maioria das UTIs ainda fazem análise qualitativa. Sabe-se também que somente a análise microbiológica não é suficiente para diagnosticar essa doença. Para um bom diagnóstico é necessário uma avaliação clínica crítica e eficaz ${ }^{10}$. Se a PAV foi diagnosticada clinicamente, a cultura será necessária para identificação do agente patogênico e assim a mesma não precisa ser analisada de maneira quantitativa ou broncoscópica ${ }^{10}$. 0 estudo de Cook e Mandell11 confirma o valor de amostras de secreção traqueal para identificar o patógeno etiológico em pacientes com PAV. Nas amostras qualitativas de secreção traqueal é raro não crescer um organismo que também será encontrado no tecido pulmonar 
usando broncoscopia. No entanto, o uso de amostras quantitativas de secreção traqueal pode melhorar a especificidade da cultura de aspirado traqueal, definindo os organismos que são susceptíveis de ser a causa de pneumonia ${ }^{13}$. Além de possibilitar a diferenciação entre colonização e infecção, uma vez que a via aérea da maioria dos pacientes em ventilação mecânica está colonizada por microorganismos potencialmente patógenos acusando resultados falso-positivo por análise qualitativa ${ }^{6}$.

Segundo Oplustil ${ }^{8}$ os agentes isolados mais comuns nas pneumonias de origem bacteriana são: Acinetobacter baumanni, Pseudomonas aeruginosa, Escherichia coli, Enterobacter spp., Staphylococcus aureus e Klebsiella spp. No presente estudo os agentes mais prevalentes foram Acinetobacter baumanni (13,6\%) e a Pseudomonas aeruginosa (13,6\%) corroborando com Opustil e com os achados de Teixeira et al.(2004) que encontraram 8,8 \% de Acinetobacter baumanni e 17,6\% de Pseudomonas aeruginosa em um estudo com 91 pacientes em ventilação mecânica. Também concordando com os estudos de Fujitani et. al., 2011 cuja incidência de Pseudomonas aeruginosa foi de 23\% em seu estudo em uma UTI geral.

No ambiente hospitalar, as fontes de maior contaminação são o ventilador mecânico, sistemas de hemodiálise, pias e materiais de limpeza ${ }^{19}$

Segundo estudos ${ }^{14,15,16}$ as bactérias de coloração Gram negativa são as mais frequentes em UTI, entre elas 0 Acinetobacter baumanni e Pseudomonas aeuriginosa, concordando com os achados dessa pesquisa (59,9\%). A característica mais importante das Gram negativas é sua versatilidade nutricional, que permite se adaptar de forma eficaz para 0 ambiente hospitalar. A sua resistência intrínseca aos múltiplos antimicrobianos facilita a aquisição de resistência aos antimicrobianos para fins terapêuticos. Esta característica explica em grande parte a capacidade que esses patógenos têm de causar surtos nosocomiais e a dificuldade de tratar essas infecções ${ }^{17}$. Porém somente a coloração Gram não é suficiente para um correto diagnóstico e prescrição de antibióticoterapia, pois tem um papel muito limitado na orientação empírica inicial da mesma em pacientes com suspeita de PAV ${ }^{14}$.

No grupo estudado houve uma presença relevante de Candida spp. $(18,18 \%)$, concordando com a pesquisa de Charles et. al., ${ }^{18}$ que encontraram $56 \%$ de colonização por Candida spp. em pacientes que apresentavam suspeita de PAV. Autores sugerem que a presença de Candida spp. dentro do trato respiratório facilita o crescimento bacteriano e assim o desenvolvimento de pneumonia, o que poderá piorar o resultado da PAV independentemente do patógeno ${ }^{18,19}$.

$\mathrm{Na}$ comparação das técnicas quanto ao número de células e leucócitos, ambas apresentaram uma boa amostra baseado nos critérios de Oplustil ${ }^{8}$ (não foi necessário repetir nenhuma coleta), apesar do coeficiente de concordância ser moderado e fraco, respectivamente. De acordo com Oplustil ${ }^{8}$, uma amostra de qualidade é aquela que segue critérios que envolvem o transporte (enviar ao laboratório à temperatura ambiente - de 20 a $25^{\circ} \mathrm{C}$ - em duas horas), a quantidade (mínimo $1 \mathrm{ml}$ ) e a aceitabilidade ( $\leq 10$ células epiteliais/campo e $\geq 25$ leucócitos/campo) da secreção. Tais critérios foram respeitados no presente estudo justificando a qualidade das amostras avaliadas.

No que se refere às bactérias, observa-se que em $77,7 \%$ dos pacientes não houve diferença nas bactérias encontradas nas técnicas 1 e 2 e em 22,3\% destes foi encontrado diferença. A concordância estatística entre as duas técnicas foi forte (coeficiente Kappa), comprovando que independente da técnica que for escolhida, a mesma não afetará o crescimento de microorganismos na amostra, do mesmo modo quanto à coloração de Gram.

Entre os germes diferentes nas duas técnicas (Tabela 3) a Serratia marcens apareceu somente na técnica 1 (com sonda cortada) em 1 paciente, porém apareceu nas duas técnicas em outro paciente sugerindo que o resultado não tem relação com a técnica. A Candida spp cresceu nas técnicas 1 e 2 em 2 pacientes e, somente na técnica 2, em outros 2 pacientes, dificultando a real interpretação quanto a sua presença. A Coagulase negativa Staphylococcus species e Staphylococcus aureus apareceram somente nas coletas pela técnica 1. Entretanto, cabe salientar que não houve associação significativa entre a técnica realizada e o germe encontrado.

A Coagulase negativa Staphylococcus species é uma bactéria que pertence à microbiota normal da pele e mucosas dos humanos ${ }^{19}$ e 0 Staphylococcus aureus é frequentemente encontrado na orofaringe de pacientes em ventilação mecânica ${ }^{20}$ sugerindo que ocorreu uma contaminação da amostra coletada pela técnica 1. Nessa técnica a parte externa da sonda, que tem contato com o biofilme interno do tubo, fica depositada dentro do frasco estéril, podendo, assim, contaminar a amostra. Tal fato, provavelmente, não ocorre na técnica 2, pois vai para a análise somente a secreção que estava em contato com o lúmen interno do tubo, não tendo esta contato com o biofilme. $O$ biofilme é um conglomerado de células bacterianas encharcados em uma matriz de exopolissacarídeos, estruturados sobre o tubo traqueal. 0 tubo é uma superfície inerte onde as bactérias podem aderir, colonizar e crescer. Forma-se, então, um micro-hábitat de bactérias que servem como uma fonte de inoculação de microorganismos nos pulmões e aumento da resistência a antimicrobianos ${ }^{20,21}$.

Quando analisamos a técnica 2, na qual a secreção é empurrada para o frasco estéril pelo fluxo que oxigênio, podemos supor que o oxigênio influencie na amostra. Sabe-se que os microorganismos são classificados em aeróbios 
estritos (exigem a presença de oxigênio) como gênero Acinetobacter ou os fungos; microaerófilos (necessitam de baixos teores de oxigênio) como o Campylobacter jejuni; facultativos (utilizam o oxigênio quando disponível, mas desenvolvem-se também em sua ausência) como Escherichia coli e várias bactérias entéricas e; anaeróbios estritos (não toleram o oxigênio) como o Clostridium tetani que só se desenvolve em tecidos necrosados, ou seja, carentes de oxigênio ${ }^{21}$. Logo o oxigênio pode ser indispensável, letal ou inócuo para os microrganismos. Geralmente, germes que não toleram o oxigênio não são encontrados na secreção traqueal, pelo próprio ambiente das vias respiratórias. Além disso, na maioria das amostras cresceram os mesmos germes, o que nos leva a crer que o oxigênio não potencializou ou inibiu o crescimento microbiano.

Considerando os resultados desse estudo, tanto a técnica que utiliza a sonda cortada, quanto a que faz uso do oxigênio pode ser utilizada para coleta da secreção traqueal de pacientes entubados ou traqueostomizados, desde que critérios rigorosos de assepsia e qualidade sejam seguidos durante a realização, conservação e transporte para o laboratório. Isso quando comparadas quanto à análise microbiológica, que foi o objetivo dessa pesquisa, todavia, outros aspectos podem ainda ser investigados em estudos subsequentes como: o custo hospitalar, o tempo gasto para a realização da técnica e a praticidade tanto para o profissional que realiza a coleta quanto o executa a análise laboratorial. Só assim poderá haver uma padronização em relação a melhor conduta para coleta.

As limitações do estudo referem-se, principalmente, ao número de amostras avaliadas microbiologicamente e à análise qualitativa realizada pelo LAC. Acreditamos que para a generalização e validação dos resultados encontrados, estudos multicêntricos devam ser efetuados, bem como a confiabilidade de cada técnica testada por análise microbiológica quantitativa.

\section{Considerações Finais}

A análise microbiológica realizada no presente estudo demonstrou que as técnicas de coleta de secreção traqueal com a sonda cortada e com o fluxo de oxigênio são equivalentes no que se refere à análise microbiológica. Logo ambas podem ser utilizadas na rotina hospitalar.

\section{Referências Bibliográficas}

1. Rodrigues EAC, Richtmann R. IRAS: infecção relacionada a assistência à saúde: orientações práticas. $1^{\mathrm{a}}$ ed. São Paulo: Sarvier; 2008.

2. Froes F, Paiva J, Amara P, Baptista J, Brum G, Bento H, et al. Documento de Consenso sobre pneumonia nosocomial. Rev Port. Pneumol. 2007; 3,;419-436

3. Khollef MH. Clinical presentation and diagnosis of ventilator-associated. Disponível em: <uww.uptodate.com> Acesso em 13 jun. 2012

4. Cubillos AF, Cifuentes M. Actualización del Consenso "Neumonía asociada a ventilación mecánica. Primera parte. Aspectos diagnósticos. Revista Chilena Infectologia 2011; 28 (2): 130-151

5. Kruger S, Frenchen D, Ewig S. Prognosis of ventilator-associated pneumonia: what lies beneath. Eur Respir Journal, 2011; 137; 486-88.

6. Estella A, Lerma S. Should the diagnosis of ventilator associated pneumonia be improve? Med Intensiva 2011; 35 (9): $578-582$

7. Fujitani S, Sun HY, Yu VL, Weingarten JA. Pneumonia Due to Pseudomonas aeruginosa: Part I: epidemiology, Clinical Diagnosis ans source. Chest 2011; 139:909-919.

8. Oplustil CP, Zuccoli CM, Toubouti NR, Sinto SF. Procedimentos básicos em microbiologia clínica. $3^{\mathrm{a}}$ ed. São Paulo: Sarvier, 2010. 9. Teixeira PJZ, Hertz FT, Cruz DB, Caraver F, Hallal RC, Moreira JS. Pneumonia associada à ventilação mecânica: impacto da multirresistência bacteriana na morbidade e mortalidade. J. bras. pneumol. [artigo na Internet]. 2004 [cited 2012 June 13] ; 30(6): 540-548. Available from:<http://www.scielo.br/scielo.php>.

10. Niedermann MS. The Argument against Using Quantitative Cultures in Clinical Trials and for the Management of Ventilator-Associated Pneumonia. Clinical Infectious Diseases 2010; 51: S93 - S99.

11. Cook D, Mandell L. Endotracheal Aspiration in the Diagnosis of Ventilator-Associated Pneumonia. Chest 2000; 117: $195 \mathrm{~S}-197 \mathrm{~S}$.

12. Ferreira H, Lala ERP. Pseudomonas aeruginosa: Um alerta aos profissionais de saúde. Rev Panam Infectol 2010;12(2):44-50. 
13. Albert M, Friedrich JO, Adhikari NKJ, Day AG, Verdant C, Heyland DK. Utility of Gram stain in the clinical management of suspected ventilator-associated pneumonia Secondary analysis of a multicenter randomized trial. Journal of Critical Care 2008; 23 (1); 74-86.

14. Cuenca FF, Cortés LEL, Baño JR. Contribución del laboratorio de microbiología en la vigilancia y el control de brotes nosocomiales producidos por bacilos gramnegativos no fermentadores. Enfermedades infecciosas y microbiologia clinica 2011; 29 (3): 40-46

15. Vandecandelaere I, Matthijs N, Van Nieuwerburgh F, Deforce D, Vosters P, et al. Assessment of Microbial Diversity in Biofilms Recovered from Endotracheal Tubes Using Culture Dependent and Independent Approaches. PLoS ONE 2012 7(6). Disponível em http://www.plosone.org. Acesso em 10 jun 2012.

16. Solh AA, Alhajhusain A. Update on the treatment of Pseudomonas aeruginosa pneumonia. J Antimicrob Chemother. 2009;64:229-38.

17. Charles PE, Dalle F, Aube H, Doise JM, Quenot JP, Aho LS. Candida spp. colonization significance in critically ill medical patients: aprospective study. Intensive Care Med 2005;31:393-400.

18. Williamson DR, Albert M, Perreault MM, Delisle MS, Muscedere J, Rotstein C, Jiang X, Heyland DK. The relationship between Candida species cultured from the respiratory tract and systemic inflammation in critically ill patients with ventilator-associated pneumonia. Can J Anaesth. 2011;58(3):275-84.

19. Amaral SM, Cortês AQ, Pires FR. Pneumonia nosocomial: importância do microambiente oral. J Bras Pneumol. 2009;35(11):1116-1124.

20. Stoodley L, Costerton JW, Stoodley P. Bacterial biofilms: from the natural environment to infectious diseases. Nature Reviews Microbiology, 2004; 2 (2): 95-108.

21. Menezes CHP, Neufeld PM. Bacteriologia e Micologia para o Laboratório Clínico. Editora Revinter. Rio de Janeiro. 2006

\section{Silvia Dubou Serafim}

Endereço para correspondência - Rua: Serafim Valandro, n 1510, apto 601, Bairro: Centro, CEP 97015-630, Cidade: Santa Maria, RS, Brasil.

E-mail: sdsilvia@hotmail.com

Lattes: http://lattes.cnpq.br/9816349456134577

Juliana Alves Souza - fisioju@yahoo.com.br

Janice Cristina Soares - janicesoares9@yahoo.com.br

Nara Lucia Frasson Dal Forno - naralfdf@gmail.com

\section{Enviado em 13 de novembro de 2013. Aceito em 17 de outubro de 2014.}

